\title{
Strategies in Interventional Radiology: Formation of an Interdisciplinary Center of Vascular Anomalies - Chances and Challenges for Effective and Efficient Patient Management
}

\author{
Strategien in der Interventionellen Radiologie: Gründung \\ eines Interdisziplinäres Zentrum für Gefäßanomalien - \\ Chancen und Herausforderungen für effektives und \\ effizientes Patientenmanagement
}

Authors

Maliha Sadick' ${ }^{1}$, Franz Josef Dally ${ }^{1}$, Stefan O. Schönberg' ${ }^{1}$, Christian Stroszczynski ${ }^{2}$, Walter A. Wohlgemuth ${ }^{3}$

Affiliations

1 Interdisciplinary Center of Vascular Anomalies, Institute of Clinical Radiology and Nuclear Medicine, University Medical Center Mannheim, Germany

2 Department of Radiology, University Hospital Regensburg, Department of Radiology, Regensburg, Germany

3 Interdisciplinary Center for Vascular Anomalies, University Hospital Halle, University Clinic and Polyclinic of Radiology, Halle (Saale), Germany

Key words

vascular anomaly, center formation, interdisciplinary, epidemiology, interventional radiology, minimally invasive therapy

received 06.01.2017

accepted 08.06.2017

Bibliography

DOI https://doi.org/10.1055/s-0043-117047

Published online: 23.8.2017 | Fortschr Röntgenstr 2017; 189:

957-966 @ Georg Thieme Verlag KG, Stuttgart · New York,

ISSN 1438-9029

Correspondence

Prof. Maliha Sadick

Interdisciplinary Center of Vascular Anomalies, Institute of

Clinical Radiology and Nuclear Medicine, University Medical

Center Mannheim, Theodor Kutzer Ufer 1-3,

68167 Mannheim, Germany

Tel.: ++49/621/3832276

Fax: $++49 / 621 / 3831910$

maliha.sadick@medma.uni-heidelberg.de

\section{ABSTRACT}

Background Radiology is an interdisciplinary field dedicated to the diagnosis and treatment of numerous diseases and is involved in the development of multimodal treatment concepts.
Method Interdisciplinary case management, a broad spectrum of diagnostic imaging facilities and dedicated endovascular radiological treatment options are valuable tools that allow radiology to set up an interdisciplinary center for vascular anomalies.

Results Image-based diagnosis combined with endovascular treatment options is an essential tool for the treatment of patients with highly complex vascular diseases. These vascular anomalies can affect numerous parts of the body so that a multidisciplinary treatment approach is required for optimal patient care.

Conclusion This paper discusses the possibilities and challenges regarding effective and efficient patient management in connection with the formation of an interdisciplinary center for vascular anomalies with strengthening of the clinical role of radiologists.

\section{Key points}

- Vascular anomalies, which include vascular tumors and malformations, are complex to diagnose and treat.

- There are far more patients with vascular anomalies requiring therapy than interdisciplinary centers for vascular anomalies - there is currently a shortage of dedicated interdisciplinary centers for vascular anomalies in Germany that can provide dedicated care for affected patients.

- Radiology includes a broad spectrum of diagnostic and minimally invasive therapeutic tools which allow the formation of an interdisciplinary center for vascular anomalies for effective, efficient and comprehensive patient management.

\section{Citation Format}

- Sadick M, Dally FJ, Schönberg SO et al. Strategies in Interventional Radiology: Formation of an Interdisciplinary Center of Vascular Anomalies - Chances and Challenges for Effective and Efficient Patient Management. Fortschr Röntgenstr 2017; 189: 957-966 


\section{ZUSAMMENFASSUNG}

Hintergrund Als Querschnittsfach widmet sich die Radiologie der Diagnostik und Therapie von zahlreichen Erkrankungen und nimmt Anteil an der Entwicklung multimodaler Behandlungskonzepte.

Methode Interdisziplinäre Ausrichtung sowie ein breites Spektrum an bildgestützten Diagnoseverfahren und spezielle endovaskuläre radiologische Behandlungstechniken sind wertvolle Bausteine, die die Radiologie fachlich dazu befähigen, ein Interdisziplinäres Zentrum für Gefäßanomalien zu etablieren.

Ergebnisse Die Kombination aus radiologischer Diagnostik und minimalinvasiver Therapie sind wesentliche Grundbausteine in der Behandlung dieser Patienten mit einer seltenen komplexen vaskulären Erkrankung, die alle Körperregionen betreffen kann und somit meist nicht von einer Fachdisziplin alleine behandelt werden kann. Im Folgenden werden Chancen und Herausforderungen für ein effektives und effizientes Patientenmanagement, die mit der Gründung eines Interdisziplinären Zentrums für Gefäßanomalien verknüpft sind, diskutiert.

Schlussfolgerung Radiologie als Querschnittsfach mit diagnostischen und minimalinvasiven therapeutischen Möglichkeiten bildet eine optimale Schnittstelle für die Betreuung von Patienten mit Gefäßanomalien. Die klinische Rolle des Radiologen ist hierbei von besonderer Bedeutung, wenn sie bereit ist, sich auch der klinischen Herausforderung zu stellen.

\section{Introduction}

February 28, 2017 marked the 10th anniversary of the international "Rare Disease Day" [1]. The approximately 30000 diseases known worldwide include 5000 rare diseases. A disease is considered rare when it affects less than 1/2000 people [2]. This includes congenital vascular anomalies comprising of vascular malformations and rare vascular tumors. The underlying malformations are highly variable in angiogenesis and vasculogenesis, can occur in any region of the body, and result in significant physical impairment depending on the degree of severity. Vascular anomalies are considered the most severe and complex amongst vascular diseases $[3,4]$. In the past the variability of this rare disease often resulted in utilization of imprecise or incorrect clinical terms for diagnosis (e.g. "hemangioma" or "angioma") and utilization of specific, medical speciality related, nomenclatures which would potentially alter the overall understanding of the disease. This fact frequently induced non-standardized treatment of vascular anomalies in the past.

Exact data regarding the number of patients with vascular anomalies is not available in Germany. According to estimates, 300000 people are diagnosed with a venous malformation [1]. Primarily very young people are affected and have to undergo complex diagnostic procedures and treatment depending on disease severity and related symptoms [5-9]. Diagnosis and the plurality of minimally invasive treatment techniques represent a major therapeutic challenge. The most recent advances in the understanding of vascular anomalies have resulted in increased interest in this disease. The demand and necessity for interdisciplinary centers for vascular anomalies providing optimized, dedicated patient care, is growing $[4,10,11]$.

Based on the number of patients with vascular anomalies requiring treatment, Germany currently has an insufficient number of specialized centers [1]. Comprehensive dedicated care of these usually young patients is important for appropriate clinical management. Effective and efficient patient care of rare diseases in particular requires an interdisciplinary setting with combined diagnostic and therapeutic approach.
Radiology offers a broad spectrum of diagnostic tools, and interventional radiology has a plurality of treatment options for these highly complex vascular diseases that very frequently can be treated minimal invasively [12-17].

The goal of this overview is to describe the role of radiology, which, as an interdisciplinary discipline, provides imaging-based diagnostic methods and dedicated endovascular treatment possibilities for effective and efficient treatment of patients with rare complex vascular diseases at an interdisciplinary center for vascular anomalies.

\section{Disease classification}

The ISSVA classification of vascular anomalies published by an international team of experts in 1992 was updated in 2014. The goal was to establish a standardized nomenclature for all medical disciplines involved in the treatment process so that diagnosis and treatment are initiated at an early point, based on the patients symptoms. The growing scientific debate regarding the underlying genetic mutations involved in the formation of vascular anomalies will allow cause-based treatment in the future [6]. Initial attempts are already being made in this regard in feto-maternal medicine [5].

Congenital vascular anomalies are rare. Vascular anomalies associated with pain and functional and cosmetic limitations should be treated to give patients a good quality of life and to avoid secondary complications. According to ISSVA, vascular anomalies are categorized as vascular tumors and vascular malformations. At this point please refer to the literature for a comprehensive tabulated list [6]. Vascular anomalies are based on a complex mesenchymal developmental disorder of one or a combination of multiple vessel types. Depending on the dominant vascular component, vascular anomalies are categorized as simple arterial, venous, capillary and lymphatic lesions or combined arteriovenous, capillary-venous, and veno-lymphatic lesions. In addition to the vascular composition, the flow characteristics of the vascular anomaly, i. e., fast flow or slow flow, is crucial for treatment $[6,7,15]$. However, there is no generally accepted 
definition for the differentiation between slow-flow and fast-flow malformations. The lack of flow voids on the T2 image in MRI is considered to be a "slow-flow" lesion by some specialists $[18,19]$. The percentage of infants and young adults up to 18 years of age at the interdisciplinary center for vascular anomalies at the University Medical Center Mannheim is $55 \%$ while the percentage of adults between 20 to 70 years of age is $45 \%$.

The new interdisciplinary classification of this rare disease usually affecting young patients demonstrates the potential spectrum of necessary interdisciplinary treatment concepts. The disease can cause diverse symptoms. Optimal care requires interdisciplinary cooperation between various medical and paramedical disciplines.

\section{Degrees of disease severity and treatment spectrum}

The clinical appearance of vascular anomalies is very diverse as are the symptoms of affected patients. In addition to localized swelling and pain, skin discoloration, ulcerations and involvement of internal organs and joints can be present, affecting almost every part of the body. According to the degree of disease severity, the treatment spectrum ranges from conservative measures such as compression therapy, analgetics and anticoagulants to minimally invasive and open surgical procedures such as sclerotherapy, embolotherapy and reconstructive surgery.

According to ISSVA, approx. $70-80 \%$ of vascular anomalies are venous malformations, approx. $5-15 \%$ are lympathic malformations and approx. $2-10 \%$ are arteriovenous malformations [5, $6]$. This distribution pattern reflects on the frequency of vascular anomaly therapy. Treatment of symptomatic slow-flow venous malformations that can manifest in every region of the body is most common. The main symptom is usually persistent pain with functional impairment due to recurrent thrombophlebitis that cannot be managed by analgetics and anticoagulants alone.

The clinical diagnosis is confirmed by radiological imaging, usually ultrasound in combination with MRI. Percutaneous sclerotherapy with polidocanol is the most frequently used treatment method [20]. In a combined approach with ultrasound and fluoroscopy, the venous malformation (VM) is punctured percutaneously and phlebography is performed. After exclusion of drainage of the contrast agent from the VM into main draining veins of the deep venous system, foamed polidocanol (Aethoxysklerol ${ }^{\circledR}$, Kreussler, Wiesbaden/Germany) is injected into the dysplastic venous convolute under fluoroscopy guidance until expulsion of the contrast agent from the VM can be documented. Depending on the size of the VM, one or more percutaneous needle positions may be necessary. The formation of the therapeutically desired fibrosis begins after 6 to 10 weeks. In addition, there is a sclerosing agent dose limit per treatment session. Therefore, large venous malformations may require multiple treatment sessions. The clinical diagnosis, treatment and course of a VM after percutaneous sclerotherapy are demonstrated on the basis of a patient case ( $\vee$ Fig. $1 \mathbf{a}-\mathbf{f})$.

Although polidocanol is not approved for sclerotherapy treatment of venous malformations, it has become established nation- ally and internationally as a medical standard for this indication [8, 21]. Polidocanol was originally approved for the treatment of varicosis. Primarily large venous, superficial epifascial and also intramuscular subfascial malformations can be efficiently treated in this manner.

A CE-certified medical product approved for sclerotherapy treatment of venous malformations is ScleroGel ${ }^{\circledR}$ (ab medica Germany $\mathrm{GmbH} \&$ Co. KG) $[22,23]$. This is a gel solution of ethanol $96 \%$ embedded in ethyl cellulose that allows longer contact time with the venous endothelium due to the high viscosity, resulting in a greater sclerosing effect. ScleroGel has a good safety profile. Because of its jellified consistency, the risk of extravasation is less in ScleroGel compared to polidocanol foam. In contrast to polidocanol, ScleroGel treatment usually requires fewer treatment sessions in order to achieve a significant sclerosing effect. This reduces treatment frequency and need for general anesthesia which is an important issue particularly in pediatric cases of venous malformations. These aspects justify the use of ScleroGel despite the high costs [22]. In case of application strictly according to the instruction for use, only a volume of $2 \mathrm{ml}$ (corresponds to one ampule of ScleroGel) can be administered per treatment, which can be a challenge in extensive venous malformations that may require higher volumes. However, the use of higher doses is now considered a proven standard. For this reason, more than one ampule of ScleroGel can be applied in a single session after appropriate informed consent of the patient.

Sclerotherapy treatment of lymphatic malformations (LM) is usually performed with Picibanil (OK-432), a lyophilized mixture of low-virulent strains of A streptococcus [24]. OK-432 is injected after $0.1 \mathrm{mg}$ Picibanil is diluted in $10 \mathrm{ml}$ of $0.9 \%$ saline. No more than $20 \mathrm{ml}$ of the OK-432 and saline compound, corresponding to $0.2 \mathrm{mg}$, should be injected per treatment. Following injection, Picibanil can trigger leukocytosis. It sometimes causes fever which usually subsides spontaneously, three to four days after intervention. In particular, macrocystic LM is an indication for minimally invasive treatment. Lymphatic malformations are percutaneously punctured under ultrasound and fluoroscopy and the communication of the individual macrocysts is demonstrated with contrast injection. After the lymphatic fluid has been tapped, the drained volume is replaced by a mixture of Picibanil and saline solution. It is recommended to treat large LMs with a pigtail catheter with side holes, which facilitates visualization of the LM and instillation of the sclerosing agent.

Arteriovenous malformations (AVM) represent the smallest group of vascular anomalies but are the most challenging vascular anomaly to treat minimal invasively because of their vascular complexity. Pretherapeutic transarterial or transvenous catheter angiography is helpful for anatomical differentiation between feeding arteries and draining veins. These fast-flow vascular anomalies typically require multiple embolization treatments until the nidus of the AVM is occluded and the AV shunt can be ligated. - Fig. 2 shows the transarterial embolization of a fastflow AVM in the finger using the liquid embolic agent ethylene vinyl alcohol copolymer (Onyx ${ }^{\circledR}$, Covidien/Medtronic, Germany) ( $\vee$ Fig. 2a-d). In addition to transarterial embolization using liquid embolic agents, percutaneous direct embolization and retrograde transvenous catheter embolization of arteriovenous 

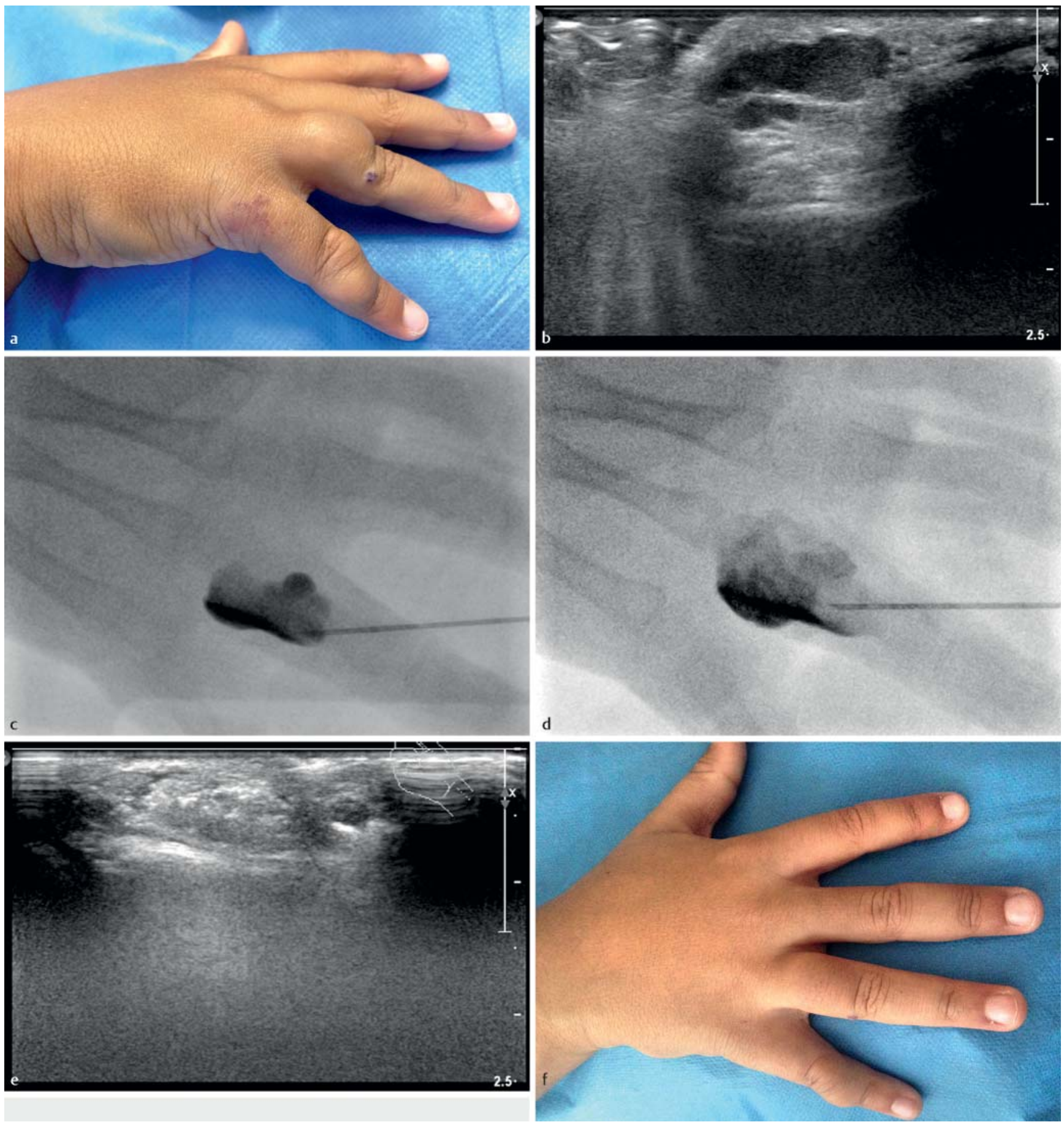

- Fig. 1 Symptomatic venous malformation (VM) located at the proximal phalanx of digit IV of the right hand in an 8-year-old patient. Swelling and pain impaired writing with the right hand a. Ultrasound demonstrates a hypoechogenic VM b. Percutaneous access to the VM and contrast injection c. Percutaneous sclerotherapy under fluoroscopy with polidocanol foam d. After sclerotherapy the VM appears hyperechogenic on ultrasound e. Outcome 8 weeks after sclerotherapy: regression of swelling of digit IV; the patient no longer complains of impaired function of the right hand. A non-symptomatic small VM with discrete swelling of digit $\mathrm{V}$ was left unsclerosed $\mathbf{f}$.

malformations are being applied more frequently now [14, 25]. Complex fast-flow AVMs often cannot be embolized via arterial access alone. In addition, there is a significant risk of migration of the embolic agent due to the fast-flow arteriovenous shunts. Transvenous retrograde access to vascular anomalies is becoming more and more popular for reaching the nidus of an AVM [25]. Ni- dus embolization via venous access can be achieved with Onyx, which is visible under fluoroscopy because of the tantalum content. This enables safe and controllable injection. The high safety profile of this substance justify the use of Onyx as a standard embolic agent for treatment of arteriovenous malformations. When anatomically possible, postinterventional resection of an occlu- 


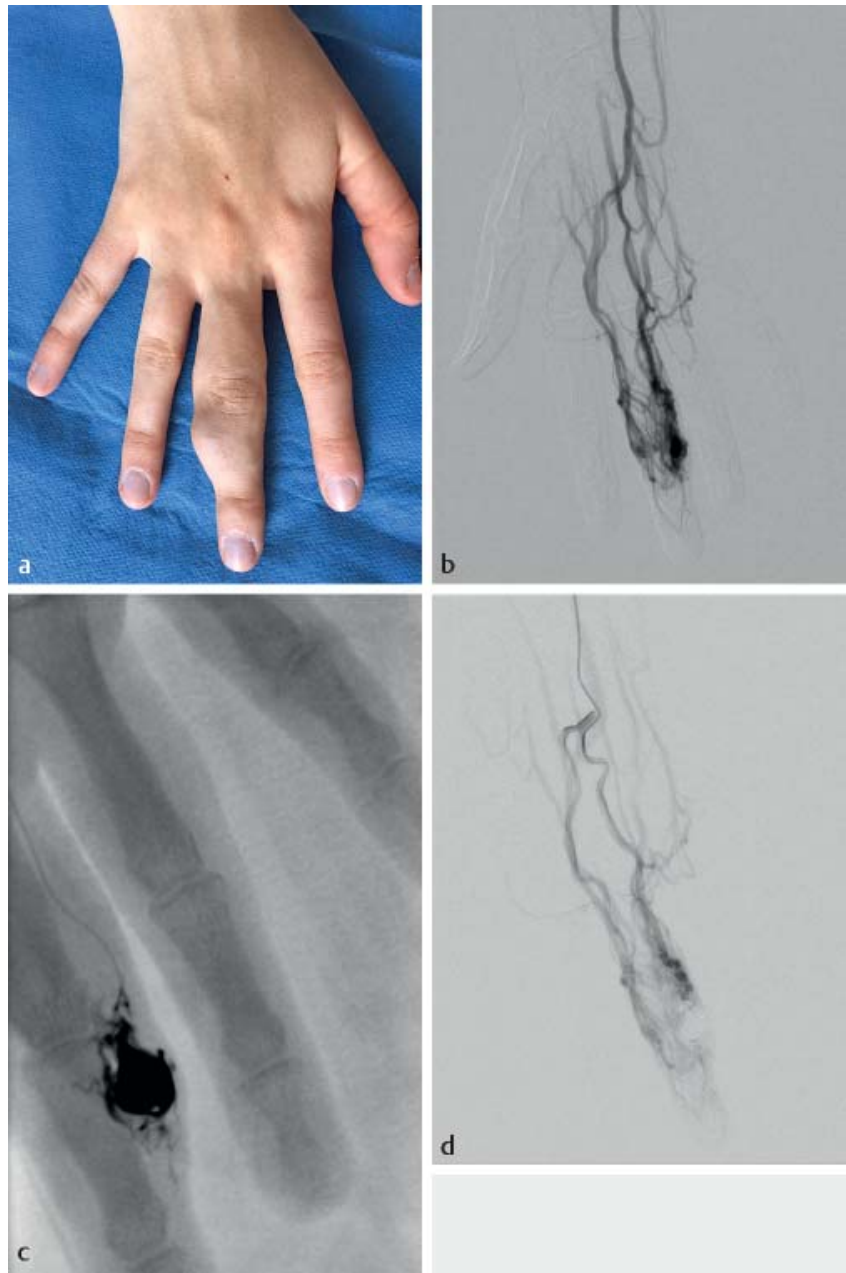

Fig. 2 Arteriovenous malformation (AVM) of third digit of the right hand in a 26-year-old patient. Macrodactylia, swelling, hyperthermia and massive pulsation were affecting the patient $\mathbf{a}$. Angiography demonstrates a fast-flow AVM of the palmar arch $\mathbf{b}$. Superselective placement of the microcatheter in the nidus of the AVM and embolization with Onyx c. Postinterventional angiography demonstrates devascularization of the nidus of the AVM of the third digit d.

ded AVM nidus is an important part of the treatment spectrum. Particularly in extensive nidus embolization close to a joint, occlusion of the AVM with Onyx may represent a functional movement restriction for the patient. A tattoo effect with discoloration of the skin after Onyx embolization of a superficial AVM can also be an indication for nidus resection.

In summary, standard treatment of the various types of vascular malformations includes several interventional procedures.

\section{Need for center formation}

With an estimated prevalence of 300000 venous malformations, which represent the most common type of vascular anomaly, Germany currently still does not have a sufficient number of centers providing specialized care for patients with vascular anomalies.
This results in increased periods of waiting time until patients are able to consult a specialist.

In light of the fact that patients are typically very young and have a chronic, often severe and untreated progressive disease, it is even more important that symptoms are analyzed and classified quickly and thoroughly so that appropriate treatment can be initiated. An interdisciplinary collaboration of different medical disciplines makes it possible to provide individually adapted patient management while meeting the necessary personnel and technical requirements. The goals of center formation are the provision of specialized diagnostic and treatment standards in an interdisciplinary setting, the optimization and coordination of the clinical workflow and the establishment of interdisciplinary scientific activity. The rarity of the disease and lack of clinical experience with vascular anomalies may result in case-based treatment of patients without the required multidisciplinary approach. Lack of exposure to vascular anomalies makes it difficult to gain diagnostic and therapeutic experience. In the case of unsuccessful treatment, the patient can be expected to seek help elsewhere in the country so that follow-up may be difficult. Since all organ systems and extremities can be affected, the small number of patients with vascular anomalies are scattered amongst various specialized clinics with limited experience and exposure to vascular malformations. This further minimizes the chances of multidisciplinary patient care.

In summary, dedicated experienced interdisciplinary centers are required for treatment of patients with vascular anomalies, a rare disease that can affect every organ in patients of all age groups.

\section{Objectives of an interdisciplinary vascular anomaly center}

The formation of an interdisciplinary center for vascular anomalies has the following goals:

- Provision of optimal patient management consisting of diagnosis, therapy, and patient care on the basis of interdisciplinary exchange and collection of clinical experience in all areas of specialization for vascular anomalies

- Application of individualized, dedicated diagnostic tools that address patient-specific symptoms and the severity of disease manifestation

- Treatment decisions based on interdisciplinary agreement and under consideration of the available minimal invasive therapeutic spectrum (therapeutic effect and side-effect/risk assessment)

- Performance of dedicated interventional radiological procedures

- Establishment of diagnostic and therapeutic quality standards

- Assurance of a high level of patient satisfaction with regional and national perception and recognition of the center

- Increase of outpatient and inpatient frequency and achievement of best patient care results

- Interdisciplinary application of all available technical and scientific resources 
- Establishment of interdisciplinary research collaborations (including scientific applications for research funding); project funding by industry and medical sciences and technology; strategic positioning of the center

- Collaboration with foundations and self-help groups (including the German Association of Vascular Anomalies [2])

Radiology is particularly suited for meeting these objectives in the context of a center formation.

\section{Role of radiology in an interdisciplinary center}

This complex disease requires a treatment mode that combines organ specific diagnosis with interventional treatment since open surgical therapy only plays a minor role in patient management. Therefore, the core competences of interdisciplinary radiology are required in this setting. However, these core competences (dedicated multiorgan diagnostic imaging and minimally invasive treatment options for the majority of affected patients) are hindered by the fact that radiology is a medical field with rare or no direct in-patient care. Therefore, a major challenge for radiology is to actively participate in clinical patient management. Direct inpatient care would enable diagnosis and therapy in one hand, preventing unnecessary loss of medical information between the individual steps of patient management. Direct clinical access to patients enables radiology to apply its broad spectrum of diagnostic and interventional tools for dedicated and optimal treatment of patients who require disease-focused care. Since only radiology performs all diagnostic procedures and minimally invasive treatment methods, it is ideally suited for managing an interdisciplinary center for vascular anomalies. Moreover, of all areas of medical specialization, radiology is most accustomed to interdisciplinary work, which represents an essential component of such a center.

Experience and competence in diagnostic radiology are a valuable part of effective and efficient patient management. High-resolution ultrasound including contrast-enhanced techniques, latest generation computed tomography scanners, multichannel magnetic resonance imaging, and catheter angiography fulfill the necessary technical requirements for diagnosing these multifactorial diseases.

Expertise in endovascular treatment techniques, including sclerotherapy and embolotherapy, are important components of treatment, considering the fact that almost $90 \%$ of all vascular anomalies can be managed by minimally invasive interventions $[15,17,25]$. The existing therapeutic spectrum is broad. It comprises sclerotherapy treatment with alcohol, polidocanol, and Picibanil (OK-432), endovascular and percutaneous laser therapy, radiofrequency ablation, transarterial, transvenous, and percutaneous embolization and even a combination of these methods $[21,26,27]$. Open surgery represents an integral part of the treatment spectrum to be offered and is used in approx. $10 \%$ of cases.

The preamble of the German Society of Interventional Radiology and Minimally Invasive Therapy (DeGIR) defines the role of interventional radiology for diagnosis and treatment using minimal- ly invasive methods [28]. In relation to treatment success and complication rates, minimally invasive, image-guided treatment methods are at least equal to, and often even superior, to open surgical methods in this field. The Academy for Training and Development of the German Radiological Society supports the activities of the DeGIR. After completion of a training program, a core competence in "diagnostic radiology" entailing fundamental competence in interventional methods can be expanded to "interventional radiology". A two-step certification program of the DeGIR enables radiologists to undergo a training in dedicated interventional procedures. The basic board certification for Interventional Radiology can be upgraded to a special certificate with integrated modules for dedicated interventional skills, corresponding to a clearly defined area of specialization in Interventional Radiology. Certification is received only upon proof of the corresponding expertise entailing sufficient practical clinical experience documented on the basis of a minimum number of independently performed interventional procedures. Moreover, currently a dedicated examination consisting of multiple choice questions and an oral practical case-based examination must be taken in order to achieve the certificate for Interventional Radiology. Continuing education in Interventional Radiology has also become a prerequisite for certification [28-30]. Centers with significant expertise in interventional diagnosis and treatment have the opportunity to become a certified DeGIR training center [30]. Thus, a quality-assured standard, both for diagnosis and treatment, is ensured and maintained which would be highly beneficial for an interdisciplinary center for vascular anomalies.

Regardless of the standard of expertise available in professional societies, dedicated interdisciplinary cooperation amongst all medical disciplines that are treating patients with vascular anomalies, supports the treatment concept. Close collaboration with pediatrics, pediatric surgery, otolaryngology, and head and neck surgery, dermatology, plastic and reconstructive surgery, vascular surgery, and hemostaseology is an essential part of patient care. It has to be mentioned that the necessary competence for the formation of a center for vascular anomalies can be acquired and expanded by internships and clinical training at designated centers nationally and internationally. The national internship network of DeGIR with the integration of European certification initiatives in Interventional Radiology provides another important educational component, particularly with respect to the diagnosis and treatment of rare diseases [30].

\section{Concrete requirements regarding the organizational structure}

The equipment of an interventional radiological center should include a multimodal imaging facility, space for recording patient medical history, clinical examination rooms and ultrasound units. Conference rooms with integrated hardware and software installations should be available that can be utilized for interdisciplinary case conferences. This set-up would provide an optimal platform for outpatient consultation, coordination of inpatient admission and interventional treatment planning ( $\triangleright$ Fig. 3). 


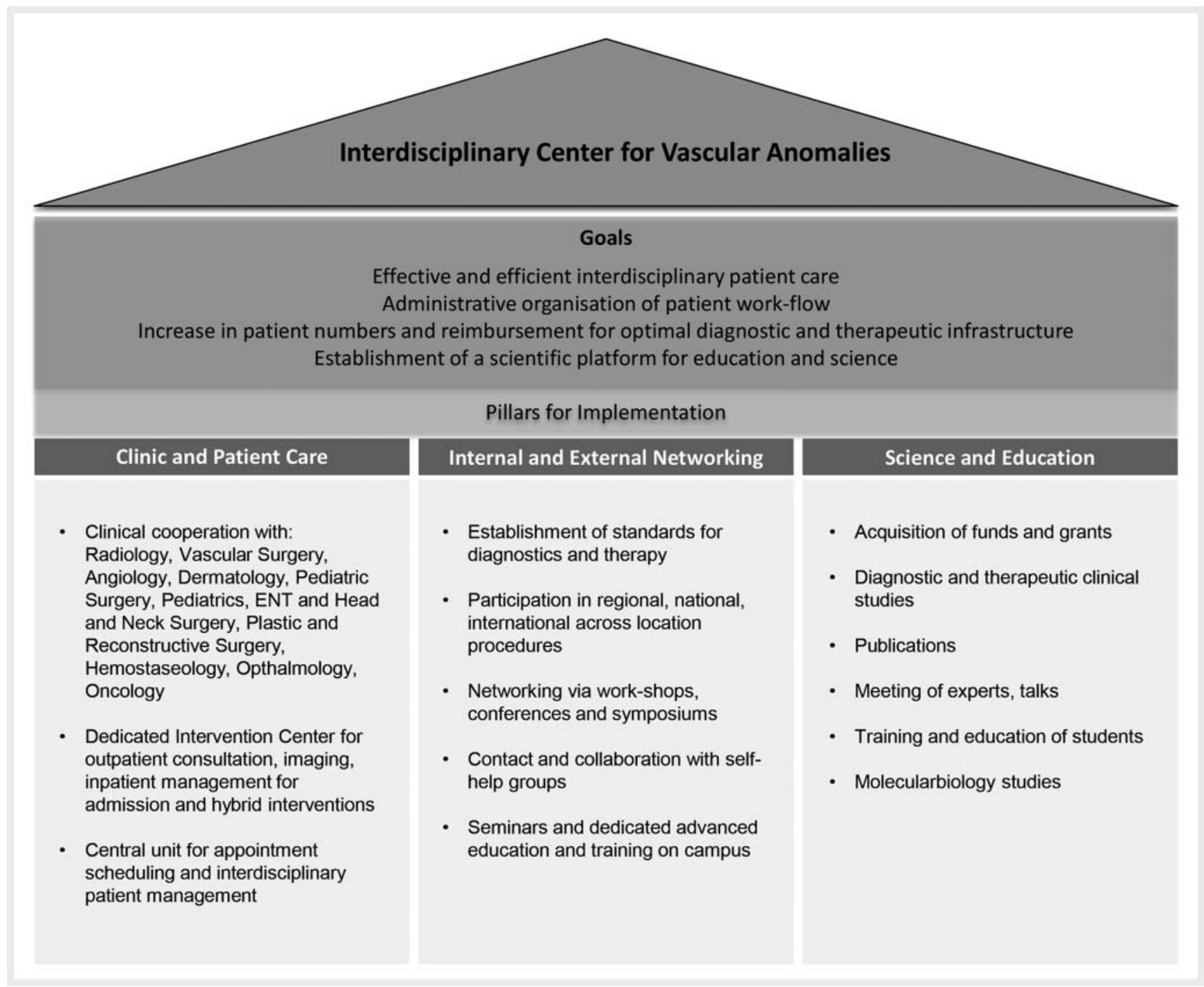

- Fig. 3 Components of optimal and efficient interdisciplinary patient care.

Patients with vascular anomalies are primarily young and have no or minimal comorbidities. And yet these patients differ greatly from patients with other vascular disease and related cardiovascular risk profile, such as peripheral arterial disease that is associated with coronary artery disease, arterial hypertension, and diabetes mellitus and its sequelae, such as diabetic nephropathy. In case of required minimal invasive treatment, it can be assumed that these patients will not exceed a hospital stay of 3 to 4 days and usually they do not require intensive unit. Sclerotherapy treatment of vascular anomalies can be scheduled as an elective intervention. This allows early and advanced inpatient scheduling and permits optimization of the admission process and allocation of inpatient beds in hospital, respectively.

A certain number of patients, in particular infants and small children, require anesthesiological monitoring during treatment and also postinterventional monitoring, which has to be taken into consideration, too.

Patient acquisition, treatment and postprocedural care are essential and time-consuming components of the organizational structure of an interdisciplinary center for vascular anomalies. Therefore, physicians must be supported by full-time administrative personnel. Besides providing dedicated clinical diagnosis and therapy, an interdisciplinary center for vascular anomalies has to create public awareness for its patient care with an interdisciplinary focus. This includes efficient patient scheduling, fast patient reporting and coordinate work with self-help groups, foundations, and scientific committees. Effective networking and interdisciplinary collaboration are important components of successful interdisciplinary patient care. This includes coordination of case conferences, symposiums, and conventions, publication of scientific articles, and fund raising for research and teaching in the field of vascular anomalies.

Molecular biological in vitro and in vivo studies of vascular anomalies with immunohistochemical and histopathological analyses are time-, personnel- and cost-intensive but are integral parts of effective and efficient patient management. All components of optimal and efficient interdisciplinary patient care are summarized in an illustration ( $\triangleright$ Fig.3). An interdisciplinary cen- 
ter for vascular anomalies requires an infrastructure which includes primary nursing and case management. Only with a combination like this, high-quality patient care can be achieved. Thus, the medical personnel at such a center should have additional qualifications including knowledge of wound management, pain management, and psychology. Professional know-how about inpatient admission procedures is also important. Due to the current shortage of dedicated centers for patients with vascular anomalies, it can be assumed that the number of patients will increase, once a vascular anomaly center has been established. Hospital administration would usually provide structural and logistic support in the initial phase of center formation in order to provide further assistance and additional personnel, once the center has been established and has started functioning successfully. Sufficient coverage of the indicated minimally invasive treatments in the DRG system should allow reimbursement for the additionally required personnel. Sufficient staffing is necessary to be able to offer adequate patient care.

\section{Radiological patient management}

In the case of rare diseases such as vascular anomalies, patients are often referred with an incorrect diagnosis or no diagnosis. A diverse number of already performed treatments and resulting extensive patient medical histories must be taken into consideration. The interdisciplinary center for vascular anomalies plays an important role in patient management with respect to early correct diagnosis and treatment which has far-reaching consequences for the overall disease course, quality of life, and socioeconomic factors. The complexity of symptomatic vascular anomalies usually requires multifactorial treatment which can be time-consuming. Patients benefit greatly from effective collaborations between various fields of specialization. The network should ensure an interdisciplinary exchange of knowledge in weekly to monthly case conferences. The discussion should include presentation of complex cases of vascular anomalies that can only be treated successfully by an expert with vast experience due to frequent exposure to vascular anomalies. Short time intervals between outpatient presentation, diagnosis and treatment would contribute to effective and efficient patient management.

A central administrative office for scheduling appointments, diagnostic procedures and outpatient follow-up should also be integrated. A coordination office that prepares an electronic patient file with relevant medical history, preliminary findings, and imaging results in advance, facilitates patient management, too. Following patient presentation and consultation, a dedicated report with diagnosis and therapy recommendations is provided for the referring physician as well as the patient.

The treating interventional radiologist should be in charge of the postprocedural examination as well as the follow-up. Radiological diagnostic and therapeutic facilities enable individualized and dedicated patient management.

\section{Inpatient care at a hospital}

Complex vascular anomalies require specialized procedures. Particularly in cases of complex arteriovenous malformations, which might be complicated due to underlying symptoms, such as ulcers and superinfection, patients benefit from inpatient care. In specialized units, qualified medical and paramedical professionals can attend to wound and pain management.

A radiology department with inpatient beds has the advantage of providing highly specialized, high-quality procedures. Usually diagnosis and treatment goes hand in hand in dedicated units with subspecialized staff. Interventional Radiology is involved in patient care during every stage of treatment. The utilization of resources can also be optimized since patients are admitted electively, based on severity of the vascular anomaly and necessity for interventional treatment. This has a corresponding effect on the diagnosis related groups revenue that can be directly allocated to radiology as a department with inpatient facilities [31]. Service-based reimbursement for inpatient management in radiology can be invested in the maintenance and expansion of a specialized infrastructure for minimally invasive diagnosis and treatment. At this point it needs to be mentioned that a radiology department with inpatient management has to fulfill certain requirements before it can live up to the expectation of a well organized center for vascular anomalies, particularly under current conditions with shortage of personnel and limited economic resources. In addition to provision of trained staff for inpatient care, quality management in the fields of hygiene, transfusion, and DRG coding must be maintained.

Therefore, inpatient care of patients with vascular anomalies at a joint interdisciplinary unit can be an useful alternative to a radiology department with inpatient management. Factors, such as a lack of comorbidities in young patients, elective treatment planning, and the possibility of optimization of inpatient occupancy make vascular anomalies a special and significant topic in patient care.

\section{Economic implications of center formation}

In contrast to cardiopulmonary and oncological diseases that usually require very urgent management, most patients with vascular anomalies can be treated electively and therefore allow elective scheduling. Preliminary outpatient appointment management and inpatient care can be planned before patients present in the vascular anomaly center. Patients may also submit external medical reports and imaging findings at the time of outpatient presentation. This approach preserves internal resources for further treatment planning steps. Diagnosis, treatment and postinterventional follow-up (analgetics, compression therapy, bed rest) are standardized so that documentation for the Medical Service of the health insurance funds is simplified and feasible.

In the ICD-10 diagnosis catalog, chapter XVII "congenital malformations, deformities, and chromosomal anomalies" addresses vascular anomalies [32]. The following codes are primarily considered for diagnostic coding of patients with vascular anomalies: 
- Other specified congenital fistulas of circulatory system (Q28.81)

- Other unspecified congenital malformations of circulatory system (Q28.88)

- Congenital malformation of circulatory system, unspecified (Q28.9)

- Congenital malformation of peripheral vascular system, unspecified (Q27.9)

A majority of treatments for vascular anomalies are German Procedure Classification procedures 8-836.9 D (percutaneous transluminal vascular intervention - selective embolization with fluids - vascular malformation) in combination with 8-83b.20 (additional information regarding material, type of fluid for selective embolization, ethylene vinyl alcohol) and 8-836.9 D (percutaneous transluminal vascular intervention - selective embolization with fluids - vascular malformation) in combination with 8 -83b.22 (additional information regarding material, type of fluids for selective embolization, ethylene vinyl alcohol copolymer) (German Procedure Classification catalog 2016) [33].

Considering the fact that venous malformations are the most common vascular anomaly (over $70 \%$ of cases) and that they are typically treated with aethoxysklerol, this sclerotherapy treatment is encoded under F59B in the DRG catalog with a current case weight of 1.84 . Reimbursement is very feasible in light of the low material costs for sclerotherapy [34]. In cases in which the medical use of expensive embolization materials is indicated, reimbursement might be less feasible.

The elective scheduling of vascular anomaly treatment, lack of comorbidities in young patients and feasibility of reimbursement of venous malformation treatment indicate that effective and efficient patient care can be realized at an interdisciplinary center for vascular anomalies.

\section{Conclusion}

The need for patient care in vascular anomalies remains to be a challenge nationally and internationally. Due to the complexity and rarity of the disease, diagnosis and treatment should be performed at an interdisciplinary center with the necessary expertise.

Radiology as an interdisciplinary field with diagnostic and minimally invasive therapeutic tools is an optimal interface for an interdisciplinary center for vascular anomalies. Moreover, of all areas of specialization, radiology is most accustomed to interdisciplinary work, which represents an essential component of such a center.

\section{Conflict of Interest}

The authors declare that they have no conflict of interest.

\section{References}

[1] http://www.rarediseaseday.org/country/de/germany (letzter Zugriff am 07.01.2017)

[2] http://www.angiodysplasie.de/de/ (letzter Zugriff am 07.01.2017)
[3] Wojcicki P, Wojcicka K. Epidemiology, Diagnostics and Treatment of Vascular Tumors and Malformations. Adv Clin Exp Med 2014; 23: 475 484

[4] Mattila KA, Kervinen K, Kalajoki-Helmio T et al. An interdisciplinary specialist team leads to improved diagnostics and treatment for paediatric patients with vascular anomalies. Acta Paediatrica 2015; 104: 1109 1116

[5] Tekes A, Kalayci TO, Mitchell SE. Congenital Vascular Anomalies: Classification and Terminology. In: Mauro MA, Murphy KP, Thomson KR, (eds) Image Guided Interventions. Philadelphia: Saunders; 2013: 271 - 283

[6] Wassef M, Blei F, Adams D et al. Vascular Anomalies Classification: Recommendations From the International Society for the Study of Vascular Anomalies. Pediatrics 2015; 136: e203-e214

[7] Wohlgemuth WA, Wölfle K, Schuster T et al. Hereditary Vascular Malformations: Classification, Symptoms, Diagnostics and Prognosis. Zentralbl Chir 2012; 137: $440-445$

[8] Lee BB, Baumgartner I, Berlien P et al. Diagnosis and Treatment of Venous Malformations Consensus Document of the International Union of Phlebology (IUP): Updated 2013. International Angiology 2015; 34: 97 149

[9] Clemens RK, Pfammatter T, Meier TO et al. Vascular malformations revisited. Vasa 2015; 44: 5-22

[10] Lidsky ME, Markovic JN, Miller MJ et al. Analysis of the Treatment of Congenital Vascular Malformations Using a Multidisciplinary Approach. J Vasc Surg 2012; 56: 1355-1362

[11] Chute C, Stein B, Sylvia MB et al. Perioperative care of the Vascular Anomaly Patient. Seminars Pediatric Surgery 2014; 23: $233-237$

[12] Hwang T], Girard E, Shellikeri S et al. Early Experience with X-Ray Magnetic Resonance Fusion for Low-flow Vascular Malformations in the Pediatric Interventional Radiology Suite. Pediatr Radiol 2015; PubMed PMID: 26681438 doi:10.1007/s00247-015-3485-7

[13] Lopera JE. The Amplatzer Vascular Plug: Review of Evolution and Current Applications. Semin Intervent Radiol 2015; 32: 356-369

[14] Gnannt R, Clemens RK, Pfammatter T. Transvenous Embolization of an Acquired Arteriovenous Malformation of the Arm. J Vasc Interv Radiol 2015; 26: $1585-1587$

[15] Nosher JL, Murillo PG, Liszewski M et al. Vascular anomalies: A Pictorial Review of Nomenclature, Diagnosis and Treatment. World J Radiol 2014; 28: $677-692$

[16] Teusch VI, Wohlgemuth WA, Piehler AP et al. Color-coded Perfusion Analysis of CEUS for Pre-interventional Diagnosis of Microvascularisation in Cases of Vascular Malformations. Clin Hemorheol Microcirc 2014; 58 : $183-193$

[17] Pimpalwar S. Vascular Malformations: Approach by an Interventional Radiologist. Semin Plast Surg 2014; 28: 91 - 103

[18] Greene AK, Liu AS, Mulliken JB et al. Vacular Anomalies in 5621 Patients: Guidelines for Referral. J Pediatr Surg 2011; 46: 1784-1789

[19] Weitz NA, Lauren CT, Behr GG et al. Clinical Spectrum of Capillary Malformation-Arteriovenous Malformation Syndrome Presenting to a Pediatric Dermatology Practice: A Retrospective Study. Pediatr Dermatol 2015; 32: $76-84$

[20] Heck M, Faulhaber ], Breu FX et al. Foam Sclerotherapy. Uses and Indications in Dermatology and Phlebology. Hautarzt 2012; 63: 493 - 503

[21] Uller W, Müller-Wille R, Wohlgemuth WA. Interventionelle Therapie von Gefäßmalformationen. Gefäßmedizin Scan 2015; 2: 305- 321

[22] http://www.abmedica.org (letzter Zugriff am 07.01.2017)

[23] Schuhmacher M, Dupuy P, Bartoli JM et al. Treatment ov Venous Malformations: First Experience with a new Sclerosing Agent-A Multicenter Study. Eur J Radiol 2011; 80: 366-372

[24] Smith MC, Zimmermann B, Burke DK et al. Efficacy and Safety of OK-432 Immunotherapy of Lymphatic Malformations. Laryngoscope 2009; 119 : $107-115$ 
[25] Wohlgemuth WA, Müller-Wille R, Teusch VI et al. The Retrograde Transvenous Push-through Method: a Novel Treatment of Peripheral Arteriovenous Malformations with Dominant Venous Outflow. Cardiovasc Intervent Radiol 2015; 38: 623-631

[26] Burrows PE, Mason KP. Percutaneous Treatment of Low Flow Vascular Malformations. J Vasc Interv Radiol 2004; 15: 431 - 445

[27] It P, Burrows P. Interventional Radiology in the Treatment of Vascular Lesions. Facial Plast Surg Clin North Am 2001; 9: 585 - 599

[28] http://www.degir.de/site/degir-dgnr-modul-und-stufenkonzept (letzter Zugriff am 07.01.2017)

[29] http://www.degir.de/site/degir-dgnr-modul-und-stufenkonzept/fachprüfung-stufe-2 (letzter Zugriff am 07.01.2017)
[30] http://www.degir.de/site/degir-dgnr-zertifizierung (letzter Zugriff am 07.01.2017)

[31] Witzel K, Lorenz R. Reservations Regarding the Suitability of the DRG System. Dtsch Arztebl Int 2016; 113: 250 doi: 10.3238/arztebl.2016.0250a

[32] http://www.icd-code.de/icd/code/ICD-10-GM-2016.html (letzter Zugriff am 07.01.2017)

[33] http://www.dimdi.de/dynamic/de/klassi/downloadcenter/ops/version2016/aktualisierung/p1sap2016.zip (letzter Zugriff am 07.01.2017)

[34] http://www.g-drg.de/cms/G-DRG-System_2016 (letzter Zugriff am 07.01.2017) 